\begin{tabular}{|c|c|c|c|c|c|c|}
\hline * & \multicolumn{2}{|c|}{$\alpha$} & \multicolumn{3}{|c|}{$d$} & Autorität \\
\hline I 53 & $I^{h} 22$ & ${ }^{m} I^{8}: 51$ & +25 & ${ }^{\circ} \mathrm{I}$ & 30.6 & Berl B 442 \\
\hline I 54 & I 4 & 1.69 & +25 & 28 & $5^{2.8}$ & Cbr E. $66_{3}$ \\
\hline I 55 & $\circ 5^{6}$ & 23.97 & +8 & 39 & 51.8 & Lpz II 346 \\
\hline I $5^{6}$ & $\circ 53$ & 1.96 & +8 & 32 & 57.8 & $\checkmark \quad 323$ \\
\hline I 57 & $\circ 47$ & 27.20 & +7 & $5^{2}$ & $45 \cdot 5$ & $\gg \quad 29 I$ \\
\hline $15^{8}$ & 046 & 40.69 & +7 & 40 & $57 \cdot 7$ & $\gg \quad 283$ \\
\hline I 59 & 020 & $12.8 \mathrm{I}$ & $-\mathbf{I}$ & 41 & $4 \cdot 5$ & Strb 8 I \\
\hline 160 & $2 \mathrm{r} 3$ & $4 \cdot 5^{I}$ & $+\mathrm{I}$ & 8 & $\times 5.7$ & $\mathrm{Nic}_{453}$ \\
\hline I $6 \mathrm{I}$ & $\circ 5^{6}$ & 28.83 & +10 & 6 & 10.6 & Lpz II 347 \\
\hline 162 & $\circ 43$ & 4.66 & +8 & 12 & 28.9 & $\Rightarrow \quad 260$ \\
\hline 163 & 214 & 53.30 & +7 & 6 & 47.0 & 864 \\
\hline I 64 & 212 & $6.10^{\circ}$ & +6 & 36 & 3.9 & 847 \\
\hline 165 & 2 & 44.22 & +6 & 37 & 43.4 & 816 \\
\hline 166 & I $5^{2}$ & 42.72 & +5 & 57 & 38.0 & 742 \\
\hline 167 & 27 & 39.90 & +36 & 6 & 19.1 & Bo VI $+35^{\circ} 42 \mathrm{I}$ \\
\hline 168 & 20 & 18.42 & +36 & I I & $5^{6.6}$ & Lu 965 \\
\hline 169 & I 57 & 26.07 & +36 & & 58.8 & $" 936$ \\
\hline 170 & I 55 & 12.64 & +36 & & 32.4 & $" 917$ \\
\hline $17 I$ & I 46 & $3^{8.69}$ & +36 & 22 & 45.8 & $» 818$ \\
\hline 172 & I $4 \mathrm{I}$ & 20.82 & +35 & $5^{6}$ & 16.8 & $\triangle 777$ \\
\hline I 73 & I $3^{6}$ & 59.19 & +35 & 43 & $5^{8.0}$ & Kü $7 \circ 2$ \\
\hline 174 & I 23 & $52 . \mathrm{I}$ & +33 & 9 & 8.2 & Lei 539 \\
\hline 175 & I 27 & 41.04 & +32 & 7 & $54 \cdot 5$ & $\$ 560$ \\
\hline
\end{tabular}

\begin{tabular}{|c|c|c|c|c|c|c|c|c|}
\hline$*$ & \multicolumn{3}{|c|}{$\alpha$} & \multicolumn{4}{|c|}{$\delta$} & Autorität \\
\hline 176 & & $22^{\mathrm{D}}$ & 56506 & + & $2^{\circ}$ & & 35.0 & Alb 677 \\
\hline 177 & 2 & 18 & 46.79 & & I & 59 & I 3. I & > 665 \\
\hline 178 & 03 & $3 \mathrm{I}$ & 22.00 & + & 7 & 42 & 24.6 & Lpz II i 81 \\
\hline I 79 & 02 & 23 & 26.76 & + & 6 & 47 & 30.0 & 134 \\
\hline 180 & 2 & 7 & 41.82 & - & 6 & 30 & 38.4 & Ott 468 \\
\hline I 8 I & I & 50 & 42.31 & - & 7 & 25 & I 2.4 & * 394 \\
\hline I 82 & 4 & 8 & 5.95 & $+3 c$ & & 52 & $45 \cdot 5$ & Lu 2160 \\
\hline 183 & 4 & $\circ$ & 23.24 & +3 & & 52 & 58.7 & $\$ 2100$ \\
\hline 184 & 4 & I 6 & 9.98 & +3 & & 46 & $3^{8.1}$ & $》 2217$ \\
\hline I 85 & $2 \mathrm{I}$ & 5 & $\mathrm{r} 9.37$ & $-I$ & & 57 & 24.6 & Cbr M. 7502 \\
\hline r 86 & 2 I & 8 & $26.1 \mathrm{I}$ & -1 & & 53 & 19.0 & 7518 \\
\hline I87 & 21 & 9 & $45 \cdot 37$ & $-I$ & & 47 & 51.9 & 7527 \\
\hline I 88 & 2 & 26 & 10.43 & +2 & & 6 & 44.5 & Berl B 755 \\
\hline 189 & 3 & $5 \mathrm{I}$ & 31.31 & +1 & & 59 & 43.6 & Berl A 1049 \\
\hline 190 & 3 & 39 & 49.29 & $+I$ & & 54 & 41.0 & $\Rightarrow 1000$ \\
\hline I $9 \mathrm{I}$ & I & 53 & 33.09 & - & I & 28 & 17.4 & $\mathrm{Nic} 391$ \\
\hline 192 & 2 & 1 & $8.6 \mathrm{I}$ & - & 1 & $2 \mathrm{I}$ & 7.1 & $\Rightarrow 421$ \\
\hline 193 & 2 & 39 & 26. I I & - & 2 & $5^{2}$ & 16.2 & Strb 661 \\
\hline I 94 & 2 & 43 & 6.45 & - & 3. & 33 & 42.6 & $" 669$ \\
\hline I 95 & 2 & 49 & I 6.34 & - & 4 & & I 3.9 & $" 69$ I \\
\hline 196 & I 5 & 6 & 39.27 & -2 & & & 5.2 & $\left.\mathrm{I}^{\mathrm{m}} \cdot 5^{1}\right), \quad 1920.45 \mathrm{vm} . \mathrm{m}$ \\
\hline
\end{tabular}

\title{
Über die Bestimmung der Länge aus einem Azimute oder einer Zenitdistanz des Mondes. Von P. Harser.
}

Die in der Überschrift genannten beiden Aufgaben, die in dieser Reihenfolge als erste und $z$ weite unterschieden werden sollen, können ohne jede Schwierigkeit durch Näherungen mit der Hilfe von Differentialformeln indirekt gelöst werden, das geschieht z. B. in Chauvenets Manual of Spherical and Practical Astronomy (Vol. I, Philadelphia r885). Man kann sie aber besser direkt lösen, wenn man die Deklination des Mondes mit Hilfe der aus zwei Mondörtern der Ephemeride berechneten Lage der augenblicklichen Mondbahnebene als Funktion seiner Rektaszension darstellt. Diese direkte Lösung habe ich für die erste Aufgabe, aber nur für diese, in der Schrift "Über geographische Ortsbestimmungen ohne astronomische Instrumente" (Ergänzungsheft Nr. $\mathbf{1}_{2} 3 \mathrm{zu}$,Petermanns Mitteilungen \&, Gotha 1897) dargelegt. Hier sollen nun die direkten Lösungen für beide Aufgaben gegeben werden; sie können beide zusammen in komplexen Gleichungen dargestellt werden, von denen sich der reelle Teil auf die erste Aufgabe, der imaginäre Teil auf die zweite Aufgabe bezieht. Bei der Trennung der beiden Teile sollen die Formeln für die beiden Aufgaben in der angenommenen Reihenfolge nebeneinander gestellt werden. Zur vollständigèn Lösung der Aufgaben gehört auch, daß untersucht werde, wie Fehler der Beobachtung und der Ephemeride, und überdies auch, wie die Veränderlichkeit der Mondbahnebene auf den Wert der Länge einwirken, damit man einerseits die Genauigkeit des Wertes der Länge beurteilen, andererseits für die Beobach- tungen und für die beiden zur Bestimmung der Lage der Mondbahnebene $z \mathfrak{u}$ benutzenden Mondörter günstige Verhältnisse auswählen könne. Was besonders die Verschiebung der Mondbahnebene durch die störende Kraft der Sonne betrifft, so habe ich für die erste Aufgabe, und nur für diese, in der genannten Schrift eine Formel für ihren Einfluß auf den Wert der Länge nur angeführt, aber nicht bewiesen, weil ein Beweis die elementare Darstellung der Schrift überstiegen haben würde. Hier soll der Beweis für beide Aufgaben mitgeteilt werden. Bei den anzustellenden Betrachtungen wird vorausgesetzt, daß die Beobachtungen bereits sowohl auf den Mondmittelpunkt, wie auf den Erdmittelpunkt reduziert worden seien, und daß man deshalb nur geozentrische Verhältnisse für den Mondmittelpunkt zu untersuchen habe.

I. Es werde die folgende Bezeichnung gewählt: Für den Beobachtungsort sei die Sternzeit der Beobachtung $\theta$, die geographische Breite $\varphi$, für den Mond sei die Rektaszension $\alpha$, die Deklphation $\delta$, der Stundenwinkel $t=\theta-\alpha$, das Azimut $a$, die Zenitdistanz $z$, der parallak tische Winkel $p$, das Argument der Deklination $u$; für die augenblickliche Mondbahnebene zur Zeit der Beobachtung sei die Rektaszension des aufsteigenden Knotens auf dem Äquator $\chi$, die Neigung gegen den Äquator $\psi$, der im Sinne der Mondbewegung genommene Positionswinkel sei $1 / 2 \pi-(q-p)$. Dann gelten für das Dreieck Pol, Zenit, Mond die Formeln

$$
\begin{gathered}
\cos z+i \cos a \sin z=(\cos t \cos \delta-i \sin \delta) e^{i \varphi} \quad \sin a \sin z=\sin t \cos \delta \\
\cos a+i \sin \varphi \sin a=(\cos p+i \sin \delta \sin p) e^{i t} \quad \cos \varphi \sin a=\cos \delta \sin p \\
(\cos \delta \mathrm{d} \alpha+i \mathrm{~d} \delta) e^{i p}=\cos \delta e^{i p} \mathrm{~d} \theta-\sin z \mathrm{~d} a-i \mathrm{~d} z
\end{gathered}
$$


Für das rechtwinklige Dreieck Mond, Knoten, Projektion des Mondes auf den Äquator bestehen die Formeln

$$
\begin{gathered}
\sin \psi e^{i(\theta-\chi)}=[\sin (q-p)+i \sin \delta \cos (q-p)] e^{i t} \quad \cos \psi=\cos \delta \cos (q-p) \\
\cos \delta e^{i p}=(\cos \psi-i \cos u \sin \psi) e^{i q} \cos \delta e^{i \alpha}=(\cos u+i \cos \psi \sin u) e^{i \chi} \\
\cos \delta \mathrm{d}(\alpha-\chi)+i \mathrm{~d} \delta=(\mathrm{d} u+i \sin u \mathrm{~d} \psi) e^{i(q-p)} .
\end{gathered}
$$

Aus den zwei ersten dieser Formeln findet man $\operatorname{tg} \delta=\operatorname{tg} \psi \sin (\alpha-\chi)$

und die letzte nimmt die folgende Gestalt an:

$$
(\cos \delta \mathrm{d} \alpha+i \mathrm{~d} \delta) e^{i p}=[\mathrm{d} u+\cos \psi \mathrm{d} \chi-i(\cos u \sin \psi \mathrm{d} \chi-\sin u \mathrm{~d} \psi)] e^{i q} .
$$

Mit Formeln der beiden Dreiecke entsteht die Gleichung

$$
(\cos a+i \sin \varphi \sin a) \cdot \sin \psi e^{-i(\theta-\chi)}+\cos \varphi \sin a \cdot \cos \psi=\sin q-i \sin \delta \cos q
$$

und daraus folgt die Formel

$$
\operatorname{tg} q / \sin \delta=\{\operatorname{tg} \psi[\cos a \cos (\theta-\chi)+\sin a \sin (\theta-\chi) \sin \varphi]+\sin a \cos \varphi\} /\{\operatorname{tg} \psi[\cos a \sin (\theta-\chi)-\sin a \cos (\theta-\chi) \sin \varphi]\} .
$$

Es mögen nun die reellen Größen $\mathfrak{p}, \mathfrak{q}$, durch deren Werte die Lage der augenblicklichen Mondbahnebene bestimmt wird, durch die Formel eingeführt werden; dann wird

$$
\begin{aligned}
& \text { wird, durch die Formel } \quad \mathfrak{p}+i \mathfrak{q}=\operatorname{tg} \psi e^{i \chi} \\
& \text { hrt werden; dann wird } \quad \operatorname{tg} \delta=\sin \alpha \mathfrak{p}-\cos \alpha \mathfrak{q} \\
& (\mathrm{d} p+i \mathrm{dq}) \cos \delta e^{-i \alpha}=\left(\mathrm{d} \psi / \cos ^{2} \psi+i \operatorname{tg} \psi \mathrm{d} \chi\right) \cos \delta e^{-i(\alpha-\chi)}=\left(\mathrm{d} \psi / \cos ^{2} \psi+i \operatorname{tg} \psi \mathrm{d} \chi\right)(\cos u-i \cos \psi \sin u)
\end{aligned}
$$

Dieser Formel entnimmt man den imaginären Teil in der Gestalt

Damit wird

$$
\mathrm{d} \mathfrak{r}=\cos \delta(\sin \alpha \mathrm{d} \mathfrak{p}-\cos \alpha \mathrm{d} \mathfrak{q})=-(\cos u \sin \psi \mathrm{d} \chi-\sin u \mathrm{~d} \psi) / \cos \psi \text {. }
$$

Nach Multiplikation mit $e^{-i q}$ folgt hieraus der imaginäre Teil

$$
\sin (q-p) \cos \delta \mathrm{d} \alpha-\cos (q-p) \mathrm{d} \delta=-\cos \psi \mathrm{d} \boldsymbol{\tau}
$$

und durch Vergleichung der 2 wei Werte von $(\cos \delta \mathrm{d} \alpha+i \mathrm{~d} \delta) e^{i p}$ entsteht die Gleichung

$$
\sin z \mathrm{~d} a+i \mathrm{~d} z=\cos \delta e^{i p} \mathrm{~d} \theta-[\mathrm{d} u+\cos \psi(\mathrm{d} \chi+i \mathrm{dr})] e^{i q} .
$$

2. Die Lösung der beiden Aufgaben wird durch die Berechnung der Werte der Größen $\mathfrak{p}$, $\mathfrak{q}$ vorbereitet; dabei soll zunächst, vorbehaltlich einer späteren Prüfung, vorausgesetzt werden, daß diese Größen fụ̈ die mäßige Anzahl von Stunden, für die äußersten Falles die Ephemeride gebraucht wird, als unveränderlich angesehen werden dürfen. Dann erlangt man für die Werte der beiden Größen $\mathfrak{p}, \mathfrak{q}$ mit Hilfe zweier Mondörter $\alpha_{1} \delta_{1}, \alpha_{2} \delta_{2}$ der Ephemerid€ nach der letzten Gleichung für $\operatorname{tg} \delta$ die künftig als erste bezeichnete Formel

$$
\mathfrak{p}+i \mathfrak{q}=\left(-\operatorname{tg} \delta_{1} e^{i \alpha_{3}}+\operatorname{tg} \delta_{2} e^{i \alpha_{1}}\right) / \sin \left(\alpha_{2}-\alpha_{1}\right) \text {. }
$$

Sie nimmt mit den Abkürzungen $\alpha_{1}=\alpha-\beta, \alpha_{2}=\alpha+\beta, \delta_{1}=\delta-\varepsilon, \delta_{2}=\delta+\varepsilon$ die folgende Gestalt an

$$
\mathfrak{p}+i \mathfrak{q}=\left(\operatorname{tg} \varepsilon / \cos ^{2} \delta \sin \beta-i \operatorname{tg} \delta / \cos ^{2} \varepsilon \cos \beta\right) e^{i \alpha} /\left(1-\operatorname{tg}^{2} \delta \operatorname{tg}^{2} \varepsilon\right) \text {. }
$$

Rücken die beiden Mondörter einander unendlich nahe, so werden $\beta, \varepsilon$ unendlich klein und ihr Verhältnis $\varepsilon / \beta$ wird gleich dem Verbältnisse $\delta^{\prime} / \alpha^{\prime}$. der Geschwindigkeiten in $\delta$ und $\alpha$, deren Werte man der Ephemeride des Nautical Almanac entnimmt; dort werden nämlich die auf $10^{\mathrm{m}}$ mittl. Zeit bezogenen Änderungen $\Delta \alpha, \Delta \delta$ für $\alpha$ in $Z$ eitsekunden, für $\delta$ in Bogensekunden angegeben. Hier und weiterhin sollen aber die Geschwindigkeiten auf die Sekunde der Sternzeit bezogen und in Einheiten des Halbmessers ausgedrückt werden, dementsprechend erhält man die Werte der Größen $\alpha^{\prime}$, $\delta^{\prime}$ nach den Formeln

$$
\alpha^{\prime}={ }_{5} \Delta \alpha / 9024.64 \quad \delta^{\prime}=\Delta \delta / 9024.64
$$

und mit ihnen findet man die Werte der Größen $\mathfrak{p}, \mathfrak{q}$ nach der künftig als zweite bezeichneten Formel

$$
p+i q=\left(\delta^{\prime} / \alpha^{\prime} \cos ^{2} \delta-i \operatorname{tg} \delta\right) e^{i \alpha} \text {. }
$$

3. Nach dieser Vorbereitung führen wir nun die folgenden Bezeichnungen ein

$\omega=\theta-\chi-1 / z \pi \quad l=\operatorname{tg} \varphi \quad m+i n=\operatorname{tg} \psi e^{i \omega} \quad f=\operatorname{tg} a \quad g=\operatorname{tg} z \quad h=\cos \varphi \cos \delta \cos t / \cos a \cos z \quad x=\operatorname{tg} t$.

Dann erhalten die Gleichungen für $\cos z, \sin z \cos a, \sin z \sin a, \operatorname{tg} q / \sin \delta$ die folgende Gestalt

$$
\begin{gathered}
V\left(\mathrm{I}+f^{2}\right)=h(\mathrm{I}+l m+l n x) \quad g=h(l-m-n x) \quad f g=h\left[V\left(\mathrm{I}+l^{2}\right)\right] x \\
\operatorname{tg} q / \sin \delta=\left[-n V\left(\mathrm{I}+l^{2}\right)+f(\mathrm{I}+l m)\right] /\left[\left(m V\left(\mathrm{I}+l^{2}\right)+f l n\right] .\right.
\end{gathered}
$$

In der letzten Gleichung ist die Wurzel positiv zu nehmen und $\cos a \sin q$ und $\cos a \sin \delta \cos q$ haben die Vorzeichen des Zählers und des Nenners des rechts stehenden Bruches.

Die drei ersten Gleichungen enthalten für die erste Aufgabe die drei Unbekannten $g, h, x$; für die zweite Aufgabe die drei Unbekannten $f, h, x$. Daß $x$ als einzige Unbekannte übrig bleibe, erreicht man für die erste Aufgabe durch die Gleichung für $(f g) / g$, für die zweite Aufgabe durch die Gleichung für $\left[g^{2}+(f g)^{2}\right] /\left[V\left(1+f^{2}\right)\right]^{2}$, und zwar ergibt sich für die erste Aufgabe die wieder mit positiver Wurzel zu nehmende lineare Gleichung

$$
x=f(l-m) /\left[V\left(\mathrm{r}+l^{2}\right)+f n\right]
$$


und für die zweite Aufgabe die quadratische Gleichung

mit den beiden Wurzeln

$$
\left(1+l^{2}+n^{2}-g^{2} l^{2} n^{2}\right) x^{2}-2 n\left[l-m+g^{2} \cdot l(1+l m)\right] x+(l-m)^{2}-g^{2}(1+l m)^{2}=0
$$

$$
x=\left(n\left[l-m+g^{2} l(\mathrm{I}+l m)\right]+v^{\prime}\left(\mathrm{x}+l^{2}\right) \cdot v^{\prime}\left\{-(l-m)^{2}+g^{2}\left[(\mathrm{1}+l m)^{2}+n^{2}\left(\mathrm{1}+l^{2}\right)\right]\right\}\right) /\left(\mathrm{I}+l^{2}+n^{2}-g^{2} l^{2} n^{2}\right) \text {. }
$$

Ist hiernach der Wert von $x$ ermittelt worden, so ergibt sich daraus der Wert von $t$, wobei $\sin t$ das Vorzeichen von $\sin a$ erhalten muß, sodann findet man den Wert von $\boldsymbol{\alpha}=\boldsymbol{\theta}-\boldsymbol{t}$ und aus der Ephemeride erhält man den dazu gehörenden Wert der Greenwicher Sternzeit $\theta_{0}$ und erlangt schließlich den Wert der westlichen Länge $\lambda$ des Beobachtungsortes von Greenwich nach der Formel

$$
\lambda=\theta_{0}-\theta \text {. }
$$

I)en für die Berechnung der Fehler erforderlichen Wert von $q$ erlangt man aus der Formel für $\operatorname{tg} q / \sin \delta$; da diese Formel aber von $f$ abhăngt, so ist sie direkt nur für die erste Aufgabe brauchbar; mit Hilfe des für die erste Aufgabe gefundenen Wertes von $x$ kann man aber $f$ durch $x$ ausdrücken und erhält so die folgende, für beide Aufgaben brauchbare Formel

$$
\operatorname{tg} q / \sin \delta=\left[-n+\left(1+l m+n^{2}\right) x /(l-m)\right] /(m+n x)
$$

in $\operatorname{der} \cos q$ dasselbe Vorzeichen erhalten muß, wie $l-m=$ $\sin z \cos q / \cos \varphi \cos \psi$.

Den für die Berechnung der Fehler gleichfalls nötigen Wert der Winkelgeschwindigkeit $u^{\prime}$ des Mondes ermittelt man nach den Formeln, die aus der letzten Gleichung für die Größe $(\cos \delta \mathrm{d} \alpha+i \mathrm{~d} \delta) e^{i f}$ mit den Werten $\mathrm{d} \chi=\mathrm{d} \mathfrak{r}=\circ$ hervorgeht, nämlich nach den Formeln

$$
\begin{gathered}
u^{\prime} \cos (q-p)=\alpha^{\prime} \cos \delta \quad u^{\prime} \sin (q-p)=\delta^{\prime} \\
u^{\prime}=V\left[\left(\alpha^{\prime} \cos \delta\right)^{2}+\delta^{\prime 2}\right] .
\end{gathered}
$$

4. Es soll nun der Einfluß von Fehlern auf den Wert der Lage und zwar zuerst derjenige des Beobachtungs fehlers bestimmt werden. Dabei betrachten wir den Wert derjenigen der beiden Größen $a, z$, der beobachtet worden ist, als richtig und werfen den Beobachtungsfehler auf die Beobachtungszeit $\boldsymbol{\theta}$. Der Fehler $\mathrm{d} \boldsymbol{\theta}$ dieser Zeit erzeugt im berechneten Werte von $\alpha$ den Fehler d $\alpha$; durch ihn wird der Mond, indem auch seine Deklination um d $\delta$ fehlerhaft wird, in seiner Bahn um $\mathrm{d} u$ verschoben, und dieser Verschiebung entspricht der Fehler $\mathrm{d} \boldsymbol{\theta}_{0}=\mathrm{d} \boldsymbol{u} / \boldsymbol{u}^{\prime}$ der Greenwicher Sternzeit. Den Wert $\mathrm{d} u=u^{\prime} \mathrm{d} \theta_{0}$ setze man nun in die Gleichung für $\sin z \mathrm{~d} a+\mathrm{id} z$ ein, die entsprechend der Annahme, daß weder die beobachteten Größen, noch die Lage der Mondbahnebene geändert werden, mit den Werten $\mathrm{d} a=\mathrm{d} z=\mathrm{d} \chi=\mathrm{dr}=0$ zu bilden ist. Dadurch ergibt sich die Formel

$$
\cos \delta e^{i p p} \mathrm{~d} \theta-u^{\prime} e^{i q} \mathrm{~d} \theta_{0}=0 \text {. }
$$

Der Fehler der Länge wird also durch die Formeln

$$
\begin{aligned}
& \mathrm{d} \lambda=\left(\cos \delta \cos p / u^{\prime} \cos q-1\right) \mathrm{d} \theta \\
& \mathrm{d} \lambda=\left(\cos \delta \sin p / u^{\prime} \sin q-\mathrm{r}\right) \mathrm{d} \theta
\end{aligned}
$$

dargestellt. In Unkenntnis des wahren Wertes von $\mathrm{d} \theta$ muß ein wahrscheinlicher Wert angenommen werden. Um diesen in

seiner Abhängigkeit ron dem Orte und der Geschwindigkeit des Mondes zu bestimmen, werfen wir nunmehr den Beobachtungsfehler, indem wir die Beobachtungszeit als richtig ansehen, auf die beobachtete der beiden Größen $a, z$. Dann wird der dem bisher angenommenen Fehler $\mathrm{d} \theta$ entsprechende Fehler $\mathrm{d} a$ oder $\mathrm{d} z$ durch die mit den Werten $\mathrm{d} \chi=\mathrm{d} \imath=0$ zu bildende Gleichung für $\sin z \mathrm{~d} a+i \mathrm{~d} z$, nämlich durch die Formel $\quad \sin z \mathrm{~d} a+i \mathrm{~d} z=\left(\cos \delta e^{i p}-u^{\prime} c^{i q}\right) \mathrm{d} \theta$

bestimmt. Die Ausdrücke $\sin z \mathrm{~d} a$ und $\mathrm{d} z$ stellen nun die Abstände des beobachteten Punktes des Mondes von dem messenden senkrechten oder wagerechten Faden zur Beobachtungszeit, also zu einer Zeit dar, als der Beobachter meinte, daß sie verschwänden. Den wahrscheinlichen Wert eines solchen Fadenabstandes, der eigentlich verschwinden sollte, habe ich schon früher als "wahrscheinlichen Fadenabstand " bezeichnet, und ich habe als Prinzip für ihn angenommen, $\mathrm{daß}$ er für einen Beobachter, für ein Instrument und für eine Art von Objekten (für Sterne einerseits und für den Mond andererseits) weder von dem Orte des Objektes, noch von der Richtung und Größe seiner Geschwindigkeit gegen den messenden Faden, noch von seiner Helligkeit, noch von der Richtung des Fadens abhänge. Dieses Prinzip des konstanten wahrscheinlichen Fadenabstandes soll auch hier wieder angenommen werden. Der wahrscheinliche Fadenabstand werde nun für den Mond mit $\varepsilon$ und allgemein der wahrscheinliche Fehler einer beliebigen Größe $x$ mit $\varepsilon_{x}$ bezeichnet. Dann gelten die Formeln

$$
\varepsilon_{\theta}=\varepsilon /\left(\cos \delta \cos p-u^{\prime} \cos q\right) \quad \varepsilon_{\theta}=\varepsilon /\left(\cos \delta \sin p-u^{\prime} \sin q\right)
$$

und dementsprechend wird

$$
\varepsilon_{\lambda}=\varepsilon / u^{\prime}|\cos q| \quad \varepsilon_{\lambda}=\varepsilon / u_{i}^{\prime} \sin q \mid .
$$

Um genaue Werte der Länge zu erhalten, hat man demnach bei der Beobachtung für die erste Aufgabe große Werte, für die zweite Aufgabe kleine Werte von $|\cos q|$ zu erstreben; im ersten Falle schneidet die Mondbahnebene den Höhenkreis nahezu senkrecht, im zweiten Falle aber unter einem kleinen Winkel. Der Wert des unbeeinflußbaren Vergrößerungsfaktors $r / u^{\prime}$ liegt zwischen 23 und $3 \mathrm{I}$.

5. Zur Untersuchung des Einflusses des Ephemeridenfehle rs auf den Wert der Länge übergehend, betrachten wir zuerst, wie Fehler der Größen $\mathfrak{p}, \mathfrak{q}$, gleichviel welchen $\mathrm{U}_{\mathbf{r}}$ sprunges, den mit ihnen berechneten Wert von $\alpha$ verändern. Indem die Beobachtungen dabei als unverändert $z$ betrachten sind, gilt die erste Gleichung für $(\cos \delta \mathrm{d} \alpha+i \mathrm{~d} \delta) e^{i p}$ mit den Werten $\mathrm{d} \theta=\mathrm{d} a=\mathrm{d} z=0$ in der Gestalt

$$
(\cos \delta \mathrm{d} \alpha+i \mathrm{~d} \delta) e^{i p}=0 \text {. }
$$

\section{Ferner besteht die Formel}

$$
\sin (q-p) \cos \delta \mathrm{d} \alpha-\cos (q-p) \mathrm{d} \delta=-\cos \psi \mathrm{d} \mathrm{r}=-\cos \delta \cos (q-p) \mathrm{d} \mathrm{r} .
$$

Aus den beiden Gleichungen ergeben sich die Werte

$\mathrm{d} \alpha=\sin p \cos (q-p) \mathrm{dr} / \cos q=\sin p \cos \psi \mathrm{dr} / \cos \delta \cos q \quad \mathrm{~d} \alpha=-\cos p \cos (q-p) \mathrm{dr} / \sin q=-\cos p \cos \psi \mathrm{d} \mathbf{r} / \cos \delta \sin q$.

Was nun den Fehler der Ephemeride betrifft, die für die beiden Mondörter $\alpha_{1} \delta_{1}, \alpha_{2} \delta_{2}$ zur Bestimmung der Größen $\mathfrak{p}, \mathfrak{q}$ und für den Mondort $a \delta$ zur Beobachtungszeit in Anspruch genommen wird, so ist man wegen der Kürze 
der Zwischenzeiten berechtigt, für die der Ephemeride hinzuzufügenden Verbesserungen $\mathrm{d} \boldsymbol{\alpha}_{1}, \mathrm{~d} \delta_{1}, \mathrm{~d} \boldsymbol{\alpha}_{2}, \mathrm{~d} \boldsymbol{\delta}_{2}, \mathrm{~d} \boldsymbol{\alpha}_{0}, \mathrm{~d} \boldsymbol{\delta}_{0}$ die Beziehungen

$$
\cos \delta_{1} \mathrm{~d} \alpha_{1}=\cos \delta_{2} \mathrm{~d} \alpha_{2}=\cos \delta \mathrm{d} \alpha_{0} \quad \mathrm{~d} \delta_{1}=\mathrm{d} \delta_{2}=\mathrm{d} \delta_{0}
$$

anzunehmen. Man bilde nun durch Differentiation der Formel

$$
p+i q=\left\{\left(\delta_{2}-\delta_{1}\right) /\left[\left(\alpha_{2}-\alpha_{1}\right) \cos ^{21} / 2\left(\delta_{1}+\delta_{2}\right)\right]-i \operatorname{tg} 1 / 2\left(\delta_{1}+\delta_{2}\right)\right\} e^{\left.i^{1 / 2} / \alpha_{1}+\alpha_{2}\right)}
$$

die Gleichung für die Verbesserungen $d \mathfrak{p}$, $d \mathfrak{q}$, führe in ihr statt der Verbesserungen der beiden ersten Mondörter die des dritten ein und lasse Glieder höheren Grades in den Differenzen der drei Größen $\alpha_{1} \alpha_{2} \alpha$ einerseits und der drei Größen $\delta_{1} \delta_{2} \delta$ andererseits neben Gliedern niederen Grades fort, dann erlangt man die Formel

$$
(\mathrm{d} p+i \mathrm{~d} q) e^{-i \alpha}=\left[\mathbf{1}-\boldsymbol{\delta}^{\prime 2} /\left(\alpha^{\prime 2} \cos ^{2} \boldsymbol{\delta}\right)\right] \operatorname{tg} \delta \mathrm{d} \boldsymbol{\alpha}_{0}+2 \boldsymbol{\delta}^{\prime} \sin \delta \mathrm{d} \delta_{0} /\left(\alpha^{\prime} \cos ^{3} \boldsymbol{\delta}\right)+i\left[\boldsymbol{\delta}^{\prime} \mathrm{d} \boldsymbol{\alpha}_{0} /\left(\boldsymbol{\alpha}^{\prime} \cos ^{2} \boldsymbol{\delta}\right)-\mathrm{d} \delta_{1} / \cos ^{2} \boldsymbol{\delta}\right] .
$$

Nach Multiplikation mit $\cos \delta$ ergibt diese Formel in ihrem imaginären Teile die Gleichung

Der reelle Teil der Formel

$$
\begin{aligned}
& \mathrm{d} \mathfrak{x}=-\delta^{\prime} \mathrm{d} \alpha_{0} /\left(\alpha^{\prime} \cos \delta\right)+\mathrm{d} \delta_{0} / \cos \delta . \\
& (\mathfrak{p}+i \mathfrak{q}) e^{-i \alpha}=\delta^{\prime} /\left(\alpha^{\prime} \cos ^{2} \delta\right)-i \operatorname{tg} \delta
\end{aligned}
$$

liefert aber die Gleichung

$$
\cos \alpha p+\sin \alpha \mathfrak{q}=\operatorname{tg} \psi \cos (\alpha-\chi)=\operatorname{tg}(q-p) / \cos \delta=\delta^{\prime} /\left(\alpha^{\prime} \cos ^{2} \delta\right)
$$

Hiermit nimmt dr die folgende Gestalt an

$$
\mathrm{d} \mathfrak{r}=-\left[\sin (q-p) \cos \delta \mathrm{d} \alpha_{0}-\cos (q-p) \mathrm{d} \delta_{0}\right] / \cos \delta \cos (q-p),
$$

und für diesen besonderen Wert von $\mathrm{d} \mathbf{r}$ gelten die folgenden Verbesserungen des berechneten Wertes von $\alpha$ $\mathrm{d} \alpha=-\sin p\left[\sin (q-p) \cos \delta \mathrm{d} \alpha-\cos (q-p) \mathrm{d} \delta_{0}\right] / \cos \delta \cos q$

Die entsprechende Verbesserung der Greenwicher Sternzeit erhält man durch die Formel $\mathrm{d} \theta_{0}=\mathrm{d} \alpha / \alpha^{\prime}$; da aber die Ephemeride noch für die Beobachtungszeit der Verbesserung $\mathrm{d} \alpha_{0}$ bedarf, so tritt ein berechneter Wert von $\alpha$ um $\mathrm{d} \alpha_{0} / \boldsymbol{\alpha}^{\prime}$ früher ein, als die Ephemeride angibt; die Verbesserung der Greenwicher Sternzeit ist also

$$
\mathrm{d} \theta_{0}=\left(\mathrm{d} \alpha-\mathrm{d} \alpha_{0}\right) / \alpha^{\prime}=\cos \delta\left(\mathrm{d} \alpha-\mathrm{d} \alpha_{0}\right) /\left[u^{\prime} \cos (q-p)\right]
$$

und, da dieser Ausdruck zugleich die Verbesserung der Länge darstellt, so wird

$$
\begin{aligned}
& \mathrm{d} \lambda=-\left(\cos p \cos \delta \mathrm{d} \alpha_{0}-\sin p \mathrm{~d} \delta_{0}\right) /\left(u^{\prime} \cos q\right) \\
& \mathrm{d} \lambda=-\left(\sin p \cos \delta \mathrm{d} \alpha_{0}+\cos p \mathrm{~d} \delta_{0}\right) /\left(u^{\prime} \sin q\right) .
\end{aligned}
$$

Ersetzt man nun in Unkenntnis des wahren Wertes der Verbesserungen der Ephemeride $\cos \delta \mathrm{d} \alpha_{0}$ und $\mathrm{d} \delta_{0}$ durch ihren für beide gleich groß anzunehmenden wahrscheinlichen Wert $\varepsilon_{0}$, so entstehen die folgenden wahrscheinlichen Werte der Verbesserungen der Länge wegen der Ephemeridenfehler:

$$
\varepsilon_{\lambda}=\varepsilon_{0} / u^{\prime}|\cos q| \quad \varepsilon_{\lambda}=\varepsilon_{0} / u^{\prime}|\sin q| .
$$

Für die wahrscheinlichen Fehler der Länge gelten also inbetreff des Beobachtungsfehlers und der Ephemeridenfehler dieselben Formeln.

6. Schließlich ist noch der Einfluß der Veränderlichkeit der Lage der Mondbahnebene auf den Wert der Länge zu untersuchen und danach zu prüfen, für welche $Z$ wischenzeiten die in 2 . gemachte Voraussetzung, daß die $\mathrm{d} \alpha=+\cos p\left[\sin (q-p) \cos \delta \mathrm{d} \alpha_{0}-\cos (q-p) \mathrm{d} \delta_{0}\right] / \cos \delta \sin q$.

Größen $\mathfrak{p}, \mathfrak{q}$ als unveränderlich betrachtet werden dürfen, berechtigt sei. Die Veränderlichkeit der Lage der Mondbahnebene entsteht durch die-störende Kraft, die die Sonne auf den Mond ausübt, und zwar durch deren zur augenblicklichen Mondbahnebene senkrechte Komponente $Z$. Wir benutzen für die Sonne in großen, für den Mond in kleinen Buchstaben die folgende Bezeichnung: die geozentrischen Entfernungen seien $R, r$; für die augenblicklichen elliptischen Bahnen seien die halben großen. Achsen $\mathfrak{A}, \mathfrak{a}$; die Exzentrizität der Mondbahn, die allein gebraucht wird, sei $e$, die in Einheiten des Halbmessers ausgedrückten mittleren Bewegungen seien $\mathfrak{R}, \mathfrak{n}$; ferner sei $\mathfrak{M}$ die Summe der Massen der Sonne, der Erde und des Mondes, $m$ die Summe der Massen der Erde und des Mondes, $k^{2}$ die Anziehungskonstante; endlich sei $B$ die geozentrische Breite der Sonne über der augenblicklichen Mondbahnebene und $S$ der geozentrische Winkel zwischen Sonne und Mond. Über die Einheiten brauchen wir, indem wir inbetreff der Zeit die Sekunde der Sternzeit festhalten, für die Entfernungen und für die Massen nichts zu bestimmen, weil sich die Formeln für den Fehler der Länge von diesen Einheiten frei gestalten. Die Striche hinter einer beliebigen Größe $x$ benutzen wir, wie wir es bereits mit einem Striche für die erste Ableitung nach der Zeit getan haben, so auch mit wachsender Zahl der Striche für die wachsenden $\mathrm{Ab}$. leitungen nach der Zeit. Die Veränderung der Lage der Mondbahnebene erfolgt dann nach den folgenden Gleichungen

$$
\begin{gathered}
\psi^{\prime}+i \chi^{\prime} \sin \psi=\left(\cos ^{2} \psi / \cos \delta\right) \gamma e^{i u} \\
r=k^{2}(\mathfrak{M}-\mathfrak{m}) \operatorname{Rr}\left[\mathrm{r} /\left(R^{2}-2 R r \cos S+r^{2}\right)^{3 / 2}-\mathrm{r} / R^{3}\right] \cos \delta \sin B /\left\{k\left[\mathfrak{m a}\left(\mathrm{r}-e^{2}\right)\right]^{1 / 2} \cos ^{2} \psi\right\} .
\end{gathered}
$$

Benutzt man aber die Formeln

$$
k^{2} \mathfrak{M}=\mathfrak{A}^{3} \mathfrak{\Re}^{2} \quad k^{2} \mathfrak{m}=\mathfrak{a}^{3} \mathfrak{n}^{2} \quad u^{\prime}=\mathfrak{n}\left(\mathrm{I}-\mathfrak{e}^{2}\right)^{1 / \mathfrak{s}}(\mathfrak{a} / r)^{2}
$$

und entwickelt man die erste Wurzel im Ausdrucke für $\gamma$ nach Potenzen von $r / R$, so nimmt er mit Beibehaltung allein des wichtigsten Gliedes die folgende Gestalt an

Nun ist

$$
\gamma={ }_{3}(\mathrm{r}-\mathfrak{m} / \mathfrak{M}) \cdot \mathfrak{R}^{2} / u^{\prime} \cdot(\mathfrak{U} / R)^{3} \cdot \cos \delta \sin B \cos S / \cos ^{2} \psi .
$$

$$
\mathfrak{p}^{\prime}+i \mathfrak{q}^{\prime}=\left(\psi^{\prime} / \cos ^{2} \psi+i \chi^{\prime} \operatorname{tg} \psi\right) e^{i \chi}=\gamma(\cos u+i \cos \psi \sin u) e^{i \chi} / \cos \delta=\gamma e^{i \alpha} \text {. }
$$

Nach dieser Formel kann man auch die Ableitungen höherer Ordnung des Ausdruckes $\mathfrak{p}+i \mathfrak{q}$ nach der Zeit bilden und damit die Änderungen ermitteln, die die Größe $\mathfrak{p}+i q \mathfrak{q}$ in einer endlichen kleinen Zeit erleidet. Wir wollen so die Größen $\mathfrak{p}_{1}+i \mathfrak{q}_{1}$ und $\mathfrak{p}_{2}+i \mathfrak{q}_{2}$ für die beiden zur Bestimmung der Werte der Größen $\mathfrak{p}, \mathfrak{q}$ ausgewählten, den Greenwicher Stern- 
zeiten $\theta_{0}+\tau_{1}$ und $\theta_{0}+\tau_{2}$ zugehörenden Mondörter auf den für unsere Rechnung erforderlichen Wert der Großße $p+i q$ zurückführen, die für die der Beobachtung entsprechende Greenwicher Sternzeit $\theta_{0}$ 'gilt. Man erhält dann die Reihen

$$
\begin{gathered}
e^{-i \alpha_{1}}=\left[1-i \alpha^{\prime} \tau_{1}-1 / 2\left(\alpha^{\prime 2}+i \alpha^{\prime \prime}\right) \tau_{1}^{2}+\cdots\right] e^{-i \alpha} \\
p_{1}+i q_{1}-(p+i q)=\left\{\gamma \tau_{1}+1 / 2\left(\gamma^{\prime}+i \gamma \alpha^{\prime}\right) \tau_{1}^{2}+1 / 6\left[\gamma^{\prime \prime}-\gamma \alpha^{\prime 2}+i\left(2 \gamma^{\prime} \alpha^{\prime}+\gamma \alpha^{\prime \prime}\right)\right] \tau_{1}^{3}+\cdots\right\} e^{i \alpha} \\
{\left[p_{1}+i q_{1}-(p+i q)\right] e^{-i \alpha_{1}}=\gamma \tau_{1}+1 / 2\left(\gamma^{\prime}-i \gamma \alpha^{\prime}\right) \tau_{1}^{2}+1 / 6\left[\left(\gamma^{\prime \prime}-\gamma \alpha^{\prime 2}-i\left(\gamma^{\prime} \alpha^{\prime}+2 \gamma \alpha^{\prime \prime}\right)\right] \tau_{1}^{3}+\cdots\right.}
\end{gathered}
$$

Der imaginăre Teil der letzten Reihe liefert die Formel

$\sin \alpha_{1}\left(p_{1}-p\right)-\cos \alpha_{1}\left(q_{1}-q\right)=\operatorname{tg} \delta_{1}-\left(\sin \alpha_{1} p-\cos \alpha_{1} q\right)=1 / 2 \gamma \alpha^{\prime} \tau_{1}^{2}+1 / 6\left(\gamma^{\prime} \alpha^{\prime}+2 \gamma \alpha^{\prime \prime}\right) \tau_{1}^{3}+\cdots$

Man multipliziere nun diese Formel mit der Reibe

$$
e^{i\left(\alpha_{2}-\alpha\right)} / \sin \left(\alpha_{2}-\alpha_{1}\right)=\left[1-1 / 2\left(\alpha^{\prime \prime} / \alpha^{\prime}\right)\left(\tau_{1}+\tau_{2}\right)+i \alpha^{\prime} \tau_{2}+\cdots\right] /\left(\tau_{2}-\tau_{1}\right) \alpha^{\prime}
$$

und füge zu dem Produkte denjenigen Ausdruck hinzu, den das Produkt annimmt, wenn man die Indices 1 und 2 miteinander vertauscht; dann entsteht die Formel

$$
\begin{gathered}
(p+i q) e^{-i \alpha}+\left(\operatorname{tg} \delta_{1} e^{i\left(\alpha_{2}-\alpha\right)}-\operatorname{tg} \delta_{2} e^{i\left(\alpha_{1}-\alpha\right)}\right) / \sin \left(\alpha_{2}-\alpha_{1}\right)= \\
\quad=-1 / 2 \gamma\left(\tau_{1}+\tau_{2}\right)-1 / 6 \gamma^{\prime}\left(\tau_{1}{ }^{2}+\tau_{1} \tau_{2}+\tau_{2}{ }^{2}\right)-1 / 12 \gamma\left(\alpha^{\prime \prime} / \alpha^{\prime}\right)\left(\tau_{1}-\tau_{2}\right)^{2}-1 / 2 i \gamma \alpha^{\prime} \tau_{1} \tau_{2}+\cdots .
\end{gathered}
$$

Die Bestimmung der Größen $\mathfrak{p}, \mathfrak{q}$ in 2. entspricht nun $\mid=8.530$. Für dieses Minimum wird, indem wir den in der Annahme, daß die rechte Seite dieser. Gleichung verschwände; man muß also den so berechneten Werten dieser Größen wegen der rechten Seite gewisse Verbesserungen $d \mathfrak{p}, \mathrm{dq}$ hịnzufügen, für die der Ausdruck $(\mathrm{dp}+i \mathrm{dq}) e^{-i \alpha}$ den durch die rechte Seite dargestellten Wert annimmt. Da nun die entsprechende Verbesserung $\mathrm{d} r$ dem Koeffizienten von $-i$ in dem mit $\cos \delta$ multiplizierten Ausdrucke gleich ist, so erhält man die Formel $\mathrm{d} x=1 / 2 \gamma \alpha^{\prime} \cos \delta \tau_{1} \tau_{2}+\cdots$.

Nach 5 gelten dann für die Verbesserung der Greenwicher Sternzeit und damit auch der Länge die Formeln

$$
\begin{aligned}
& \mathrm{d} \lambda=+1 / 2 \gamma \sin p \cos \psi \tau_{1} \tau_{2} / \cos q \\
& \mathrm{~d} \lambda=-1 / 2 \gamma \cos p \cos \psi \tau_{1} \tau_{2} / \sin q .
\end{aligned}
$$

Sekunden der Sternzeit erlangten Wert in Stunden ausdrücken $\checkmark\left|\tau_{1} \tau_{2}\right|=5^{\mathrm{h}}$. . Schließen die beiden bei der ersten Formel für die Größen $\mathfrak{p}, \mathfrak{q}$ benutzten Mondörter den Mondort der Beobachtung ein, so darf demnach der Zeitunterschied zwischen ihnen mindestens auf $10^{h}$ steigen - diese Zahl entspricht dem ungünstigsten Falle der symmetrischen Einschließung des Mondortes für die Größen $\mathfrak{p}, \mathfrak{q}-$; fallen aber die beiden Mondörter, wie bei der $z$ weiten Formel für $\mathfrak{p}+i \mathfrak{q}$ angenommen worden ist, zusammen, so darf ihre Zeit mindestens um $5^{\text {h }}$ von der Beobachtungszeit entfernt sein. Man wird aber gut tun, diese Zwischenzeit klein zu erhalten; während nämlich die Berechnung der Größen $\mathfrak{p}, \mathfrak{q}$ nach der ersten Formel für $\mathfrak{p}+i \mathfrak{q}$ stets mit 6 Dezimalen ausgeführt werden kann, ist die Berechnung des ersten Gliedes der zweiten Formel auf 5 Dezimalen beschränkt, weil die Werte für die Geschwindigkeiten im Nautical Almanac nicht genauer angegeben werden. Das erste Glied der zweiten Formel für $\mathfrak{p}+i \mathfrak{q}$ hat nun in dem Maße einen geringeren Einfluß auf den mit ihm berechneten Wert von $\alpha$, als die Zeit, für die man die Größen $\alpha, \delta, \boldsymbol{\alpha}^{\prime}, \boldsymbol{\delta}^{\prime}$ zur Berechnung der Größen $\mathfrak{p}, \mathfrak{q}$ der Ephemeride entnimmt, der Beobachtungszeit näher liegt; denn für die Beobachtungszeit selbst trägt der erste, reelle Teil der Formel

$$
(p+i q) e^{-i \alpha}=\delta^{\prime} /\left(\alpha^{\prime} \cos ^{2} \delta\right)-i \operatorname{tg} \delta
$$

$\left|\boldsymbol{\tau}_{1} \boldsymbol{\tau}_{\mathbf{z}}\right|$ mindestens erstrecken darf, wenn durch die änderlichkeit der Lage der Mondbahnebene nur eine zulässige Erhöhung des wahrscheinlichen Fehlers der Länge eintreten soll. Wir nehmen an, daß diese Erhöhung gegenüber dem unvermeidlichen Anteile des Beobachtungsfehlers höchstens auf 1 $\% \%$ steigen dürfe und setzen für die einzelnen Faktoren von d2. $/ \varepsilon_{\lambda}$ die ungünstigsten, auf den zulässigen Wert von $\left|\tau_{1} \tau_{2}\right|$ verkleinernd wirkenden, Maximalbeträge, nämlich die folgenden Werte ein: $\log \left(\mathrm{d} \lambda / \varepsilon_{\lambda}\right)=9.66 \mathrm{i}-10, \log \mathfrak{N}=3.298-10$, $\log (\mathfrak{A} / R)=0.007, \log (\sin B)=8.967-$ го, $\log (\mathrm{r} / \cos \psi)=$ $0.057, \mathrm{I}-\mathrm{m} / \mathfrak{M}=\mathrm{I}, \cos \delta=\mathrm{I}, \cos S=\mathrm{I}$ und entweder $\sin p$ $=1$ oder $\cos p=\mathrm{I}$, und für gute Beobachtungen an guten Instrumenten nehmen wir schätzungsweise $\varepsilon=I^{\prime \prime}$ oder in Einheiten des Halbmessers $\log (\mathrm{I} / \varepsilon)=5.3 \mathrm{I} 4$ an; dann wird für das Minimum des zulässigen Wertes von $\left|\tau_{1} \tau_{2}\right|$ gefunden $\log \left|\tau_{1} \tau_{2}\right|$

der allein die Geschwindigkeiten enthält, zu dem durch den imaginären Teil des Differentiales dieser Formel entstehenden Werte der Größe $d x$, die für d $\alpha$ allein in Frage kommt, nichts bei. Bei der praktischen Verwendung habe ich beide Formeln gleich gut gefunden, wenn die bei der zweiten Formel nicht erreichbaren Stellen in den Werten der Größen $\mathfrak{p}, q$ beliebig, z. B. durch Nullen, ausgefüllt werden.

Die hier ermittelte Grenze für $\tau_{1} \tau_{2} \mid$ wird übrigens in allen praktischen Fällen merkbar erhöht werden dürfen, besonders, weil bei der ersten Aufgabe $|\sin p|$, bei der zweiten Aufgabe $|\cos p|$ und bei beiden Aufgaben häufig auch $|\cos S|$ nur kleine Werte haben werden.

Sternwarte Kiel, r92 I Februar.

P. Harzer.

\section{Die erste Tafel der Logarithmen und der Antilogarithmen}

und die erste Anwendung der Mechanischen Quadratur. Von A. Wedemeyer.

Nach den Angaben der mathematischen Lehrbücher hat Neper die erste Tafel der Logarithmen und Byrg die erste Tafel der Antilogarithmen berechnet. Diese Angaben ent- sprechen nicht den Tatsachen. Die erste Tafel der Logarithmen ist von Edward Wright 1594 in $A$ new and necessarie Treatise on Narigation* veröffentlicht worden. Gerhard 